\title{
A Study on Doping Density in InAs/GaAs Quantum Dot Infrared Photodetector
}

Uk Hyun LeE*, Yong Hoon Kang, Joon Ho OuM, Sang-Jun LEE ${ }^{1}$, Moondok KIM ${ }^{2}$, Sam Kyu NoH ${ }^{1}$, Yu Dong $\mathrm{JANG}^{2}$, Donghan $\mathrm{LEE}^{2}$, Hyung Seok $\mathrm{KIM}^{3}$, Chan Hyung PARK ${ }^{3}$ and Songcheol Hong

Department of Electrical Engineering and Computer Science, Korea Advanced Institute of Science and Technology (KAIST), Daejeon 305-701, Korea

${ }^{1}$ National Research Laboratory on Quantum Dot Technology, Materials Evaluation Center,

Korea Research Institute of Standards and Science, Daejeon 305-340, Korea

${ }^{2}$ Department of Physics, Chungnam National University, Daejeon 305-764, Korea

${ }^{3}$ Department of Materials Science and Engineering, Pohang University of Science and Technology (POSTECH), Pohang 790-784, Korea

(Received September 19, 2003; accepted April 1, 2004; published August 10, 2004)

We study the influence of doping density and the resulting optimum operation voltage on the performance of quantum dot infrared photodetectors (QDIPs). The optimum operation voltage, where detectivity becomes maximum, becomes smaller as the doping density increases. This is because the optimum dark current levels are similar regardless of the doping density. We confirmed experimentally that the optimum dark current level is $\sim 5 \mathrm{~mA}$ (current density: $\sim \mathrm{A} / \mathrm{cm}^{2}$ ) for our samples. It is found that the higher doping density improves the performance in the range used in this experiment $\left(5 \times 10^{16}-5 \times 10^{17} / \mathrm{cm}^{3}\right)$. The response to a normal incident light is confirmed and the possibility of high-temperature operation of QDIP is shown. [DOI: 10.1143/JJAP.43.5199]

KEYWORDS: quantum dot infrared photodetector (QDIP), doping density, operation voltage

\section{Introduction}

In recent years, quantum dot infrared photodetectors (QDIPs), which operate by intraband transitions, have gained considerable interest as a mid/far infrared (IR) detector. $^{1,2)}$ It is expected that QDIPs have two big advantages over the conventional quantum well infrared photodetectors (QWIPs). First, it can respond to normal incident light due to the three-dimensional confinement of carriers in QDs, which is verified experimentally. ${ }^{1)}$ More importantly, it is expected to operate at near room temperature because of the high photoconductive gain and low noise, which is confirmed in some papers. ${ }^{2)}$

It is necessary to dope nearby/into QDs in order to supply carriers, which will absorb IR, into QDs since QDIPs operate by intraband transitions of carriers in QDs. An increase in the doping density will allow the supply of sufficient carriers into QDs. However, this also makes the dark current larger and increases noise. Thus, the doping density is one of the most important parameters for controlling the performance of QDIPs. However, the doping density in QDIP has not been studied systematically until now. In this paper, we study the influence of the doping density and the resulting optimum operation voltage on the performance of QDIPs, and we also confirm the possibility of room temperature operation of QDIP and its response to normal incident light.

\section{Experiments and Results}

Figure 1 shows a schematic of the n-type vertical InAs/ GaAs QDIP. The three samples with the same structure except for the doping density were grown on semi-insulating GaAs (001) substrate by molecular beam epitaxy. The IR absorption region consists of 5 periods of self-assembled InAs/GaAs QD layers. The GaAs barrier thickness is $500 \AA$, which reduces dark current and noise by suppressing the vertical tunneling of carriers. ${ }^{3)}$ A $30 \AA$ n-type (Si-doped) GaAs layer was grown followed by a $60 \AA$ undoped GaAs capping layer for all five layers so that electrons, which will

*E-mail address: luhluh@kaist.ac.kr

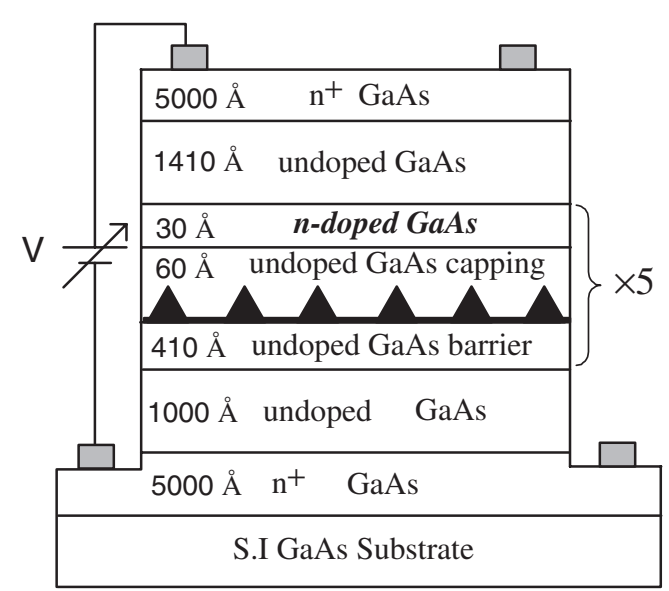

Fig. 1. Schematic of n-type vertical InAs/GaAs QDIP.

absorb the IR, are supplied into QDs. This modulation doping method will help prevent the degradation of QD quality with increasing doping density, ${ }^{4)}$ which was confirmed by PL measurements. The doping densities for each sample are $5 \times 10^{17}(\# 1), 1 \times 10^{17}(\# 2)$, and $5 \times 10^{16}(\# 3) /$ $\mathrm{cm}^{3}$. The relative doping density for each sample can be confirmed by measurements of the dark current, which increases as the doping density increases. The IR absorption region was sandwiched with $1000 \AA$ undoped GaAs buffer layers and $5000 \AA \mathrm{n}^{+}$-doped (Si: $\left.2 \times 10^{18} / \mathrm{cm}^{3}\right) \mathrm{GaAs}$ ohmic contact layers. Mesa structures with a diameter of $450 \mu \mathrm{m}$ were fabricated by standard photolithography and wet etching techniques, and then the ohmic contacts were formed with $\mathrm{AuGe} / \mathrm{Ni} / \mathrm{Au}$ alloy.

Photoluminescence (PL) measurements revealed that the QDs were well formed for all samples. Figure 2 shows normalized PL spectra of each sample at $4 \mathrm{~K}$. The PL peaks of each sample are located at 1060 (\#1), 1140 (\#2), and 1070 (\#3) nm, and the full width at half maximums (FWHMs) of PL are 120, 56, and $90 \mathrm{meV}$, respectively. Since the PL peak positions are not related to the doping density, different PL peaks are attributed to the different QD sizes and not to the 


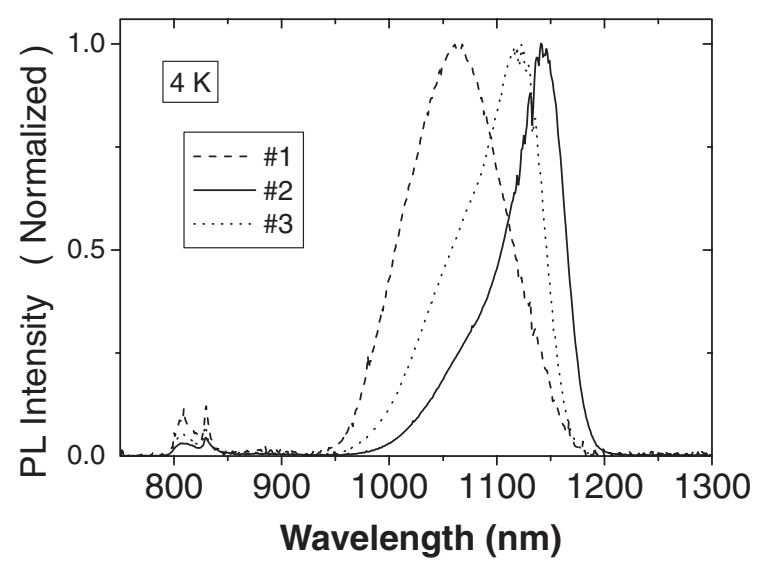

Fig. 2. Normalized PL spectra of each QDIP at $4 \mathrm{~K}$.

doping density. ${ }^{4)}$ The wavelength-integrated PL intensity (\#1:\#2:\#3 = $1: 2.9: 2.1$ ), as well as FWHM of PL, which indicates QD size uniformity, is not related to the doping density. FWHM of PL becomes larger as PL peak energy increases although the QD size uniformity is the same. ${ }^{5)}$ Thus, the QD size uniformity of the three samples is nearly similar regardless of the doping density. As a result, it is considered that QD quality is not affected by the doping density, and relatively higher/lower doping in the same structure can be achieved without degradation of QD quality.

Figure 3(a) shows photocurrent (PC) spectra of each sample at $14 \mathrm{~K}$ and Fig. 3(b) shows the temperaturedependent PC spectra of QDIP \#2. Each PC spectrum indicated by an open circle in Figs. 3(a) and 3(b) shows calibrated spectral response of QDIP \#2 at $14 \mathrm{~K}$ and at $77 \mathrm{~K}$, respectively. To calibrate the $\mathrm{PC}$ spectra, a standard MCT detector, whose spectral response is well known, was used. The PC spectra were measured under normal incident geometry using a grating monochromator, long-wavelengthpass filters, SiC IR source, preamplifier, and lock-in amplifier. Three gratings were used because of the wide spectral response, and three long-wavelength-pass filters were used to remove the harmonics for each grating. Our spectra seem somewhat noisy. This is expected to be attributable to the grating monochromator instead of the FT-IR monochromator. Moreover, the wavelength range from approximately 5 to $8 \mu \mathrm{m}$ is not an atmospheric window, in which IR absorption by air is very small. ${ }^{6)}$ However, the observed PC spectra always have similar envelopes for each sample under the same condition. For all samples in Fig. 3(a), the PC peak intensity is more than $100 \mathrm{nA}$ and the spectral response is from approximately 2 to $9 \mu \mathrm{m}$ at $14 \mathrm{~K}$. From this result, we confirm that QDIPs respond well to normal incident light. The PC shapes are complex and different from each other. However, the dip at $4.3 \mu \mathrm{m}$, which is well known to be due to atmospheric absorption, ${ }^{6)}$ was observed for all samples.

In Figs. 2 and 3(a), the PC peak energy of each sample increases relatively as the ground state (GS) PL peak energy increases. GS PL peak energy corresponds to the energy difference between electron GS in the conduction band and hole GS in the valence band, and the PC peak energy by GS-to-continuum transitions of electrons in the conduction band corresponds to the energy difference between GS and

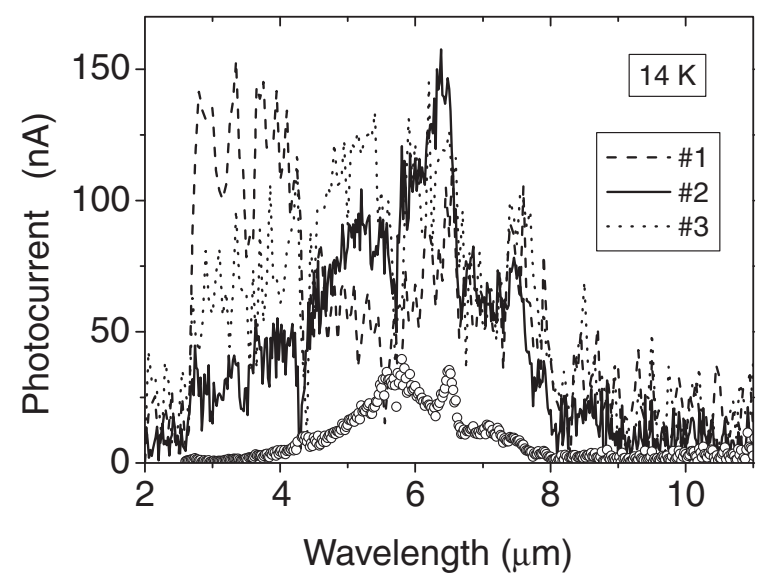

(a)

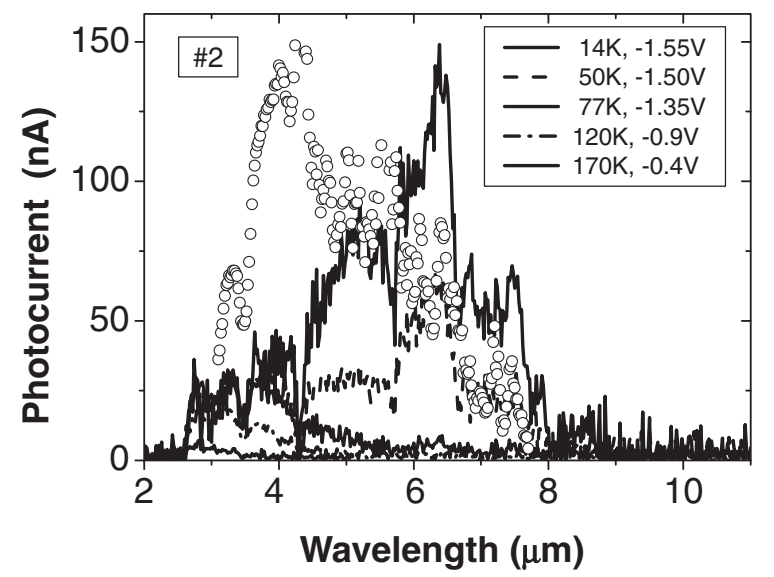

(b)

Fig. 3. (a) Photocurrent spectra of each QDIP at $14 \mathrm{~K}$. The open circle indicates calibrated spectral response of QDIP \#2 at $14 \mathrm{~K}$. (b) Temperature-dependent photocurrent spectra of QDIP \#2. The open circle indicates calibrated spectral response of QDIP \#2 at $77 \mathrm{~K}$.

the continuum of electrons in conduction band. Thus, if the PC of each sample is generated by only GS-to-continuum transitions of electrons in the conduction band, the PC peak energy should decrease as the GS PL peak energy increases. However, our results are opposite. Thus, our PC does not originate from only the GS-to-continuum transitions of electrons in the conduction band. There should be other transitions, such as GS to excited states (ES), ES to higher $\mathrm{ES}$, and ES-to-continuum transitions of electrons in the conduction band.

The temperature-dependent PC spectra of QDIP \#2 in Fig. 3(b) show that our PC has two origins: one is very sensitive to the temperature and the other is not. This temperature tendency is shown in all samples. From the calibrated PC spectrum at $77 \mathrm{~K}$ in Fig. 3(b), a relatively short wavelength PC peak, which is relatively insensitive to the temperature, is located at approximately $4 \mu \mathrm{m} \quad(\sim 310$ $\mathrm{meV})$. The other peak, which is very sensitive to the temperature, is located at approximately $6 \mu \mathrm{m}(\sim 200 \mathrm{meV})$ from the calibrated PC spectrum of QDIP \#2 in Fig. 3(a). Since the PC peak energy by the GS-to-continuum transitions of electrons in the conduction band is larger than that by other intraband transitions, the relatively higher energy PC around $4 \mu \mathrm{m}$ will originate from GS-to-continuum 
transitions of electrons in the conduction band, and the PC around $6 \mu \mathrm{m}$ will be attributed to QD ES-related transitions (ES to continuum or ES to higher ES) since this PC intensity is very sensitive to temperature. The ES-related PC intensity is more sensitive to temperature than the GS-related one since the electron density in ES is more sensitive to temperature than that in GS due to the difference in the thermal activation of the carrier in QD. ${ }^{7)}$ The first ES-related $\mathrm{PC}$ was also shown in ref. 3. As a reference, the QD average size in QDIP \#2 was $18 \times 5.2 \mathrm{~nm}$ (lateral $\times$ height), which was obtained by cross-sectional transmission electron microscopy.

The PC in the longer wavelength decreases more rapidly as the temperature increases, as shown in Fig. 3(b). This can be understood with the effect of thermal activation as follows. Electrons have to exist in QD for the operation of QDIP since QDIP operates by intraband transitions. However, electrons in QD escape thermally into the barrier as temperature increases. Thus, the thermal escape of the captured electrons makes PC signals very small. Because the energy difference between electron states in QD and the GaAs barrier potential in the conduction band is smaller, which corresponds to the lower energy (longer wavelength) in $\mathrm{PC}$, the thermal activation of the electrons becomes easier. ${ }^{7)}$ This tendency is valid regardless of the origin of PC. Thus, the PC around $6 \mu \mathrm{m}$ decreases more rapidly than that around $4 \mu \mathrm{m}$ as the temperature increases. Also, the PC at relatively longer wavelength decreases more rapidly with increasing temperature regardless of the PC peak position. As a result, it is difficult for the mid/far IR detectors to operate at higher temperature because their operation wavelength becomes longer, ${ }^{8)}$ and the thermal activation of captured carriers must be carefully studied to achieve hightemperature operation of QDIP.

Thermal activations of carriers are also observed in temperature-dependent PL spectra of QD. ${ }^{9)}$ Figure 4 shows temperature-dependent PL spectra of QDIP \#2. To compare the shape of PL spectra, PL peaks are normalized and set to $0 \mathrm{meV}$. As the temperature increases, PL signals at the relatively higher energy become smaller while the lower energy PL shape is maintained. This is also attributed to the thermal activation effects of captured carriers. ${ }^{7)}$ While the $\mathrm{PL}$ is generated by interband transitions, the PC is generated by intraband transitions of electrons in the conduction band

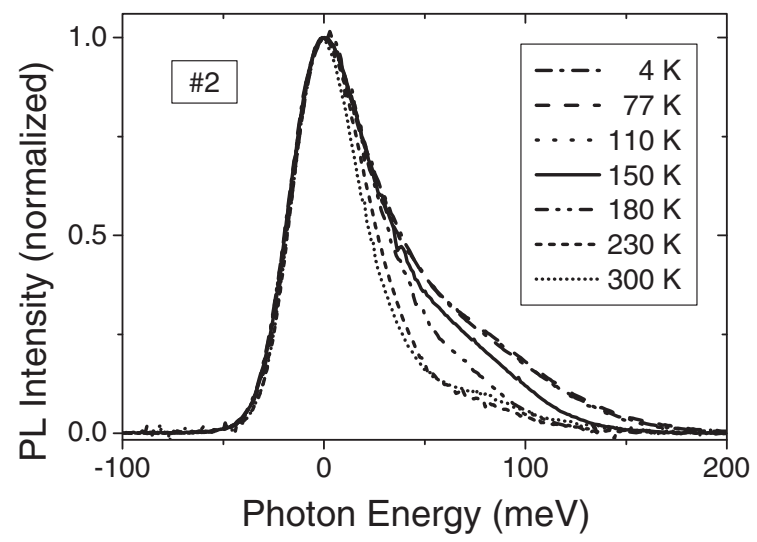

Fig. 4. Temperature-dependent PL spectra of QDIP \#2. To compare the shape of PL spectra, PL peaks are normalized and set to $0 \mathrm{meV}$.

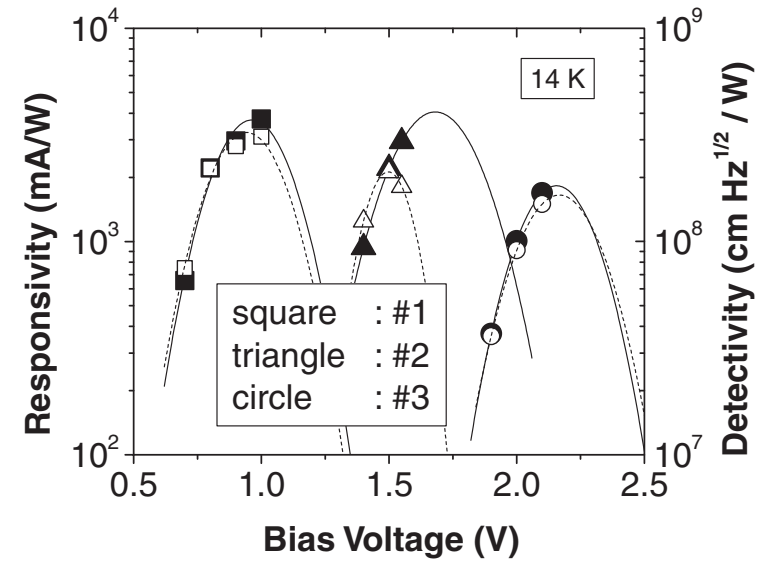

Fig. 5. Bias-dependent peak responsivity (solid symbols) and detectivity (open symbols) of each sample at $14 \mathrm{~K}$. Solid lines and dashed lines show extrapolation with second-order polynomial fitting.

and carrier transports. Thus, it cannot be expected that all results in PL exactly correspond to those in PC. However, it is clear that temperature dependence of the shapes of both PC and PL spectra can be explained by the thermal activation of carriers.

Figure 5 shows peak responsivities and detectivities of each sample as a function of bias voltages at $14 \mathrm{~K}$. The peak responsivity was obtained by calibration with a standard MCT detector, whose responsivity as a function of wavelength is well known. To obtain the detectivity, noise spectral densities (NSD, unit: $\mathrm{A} / \mathrm{Hz}^{1 / 2}$ ) were measured using a DSP lock-in amplifier at the internal modulation frequency of $5 \mathrm{kHz}$ under dark condition. The maximum responsivity is more than $1 \mathrm{~A} / \mathrm{W}$ and the maximum detectivity is $\sim$ mid $10^{8} \mathrm{cmHz}^{1 / 2} / \mathrm{W}$ for all samples. Responsivities are quite large. This originates from the large photoconductive gain due to the low capture probability of the mobile carrier. Photoconductive gain, which is obtained by $1 / 4 \mathrm{e} \mathrm{NSD}^{2} /$ dark current, ${ }^{10)}$ is more than 1000 for all samples at each optimum operation voltage. However, the detectivity is relatively small due to the large noise despite the large responsivity. It is considered that the large photoconductive gain and the large noise originate from the low capture probability of carriers. ${ }^{11)}$ This is due to the small lateral coverage of QD. This makes the lifetime of excited carriers longer and increases the dark current.

Peaks of responsivity/detectivity with respect to the bias voltage are not shown in Fig. 5 since we are not able to measure the PC data with the relatively higher bias voltage in each sample because of the current limit of the preamplifier. However, from the rough extrapolation using the second-order polynomial function, we found that the maximum detectivity increases very slowly as the doping density increases around our doping range. Reference 12 reveals that the relation between detectivity and doping becomes $D^{*} \sim\{1-\exp (-q)\} /\{\operatorname{sqrt}(\exp (q)-1)\}$. Here, the dimensionless parameter $q=$ doping concentration/constant. Therefore, the optimum doping level for detectivity exists, and detectivity has a broad peak with respect to the doping near the optimum doping level, that is, detectivity is not so sensitive to the doping density. Photoconductive gain is not related to the doping, and quantum efficiency and 
noise are strongly dependent on the doping density. However, the ratio of quantum efficiency to noise is almost independent of the doping density near the optimum doping level. From the fitting of our data using the above relation between detectivity and doping density, it is found that the doping density of QDIP \#1 is near the optimum doping level. It is also expected that the detectivity may be improved in QDIP \#1 by a slightly higher doping density.

The optimum operation voltage becomes smaller as the doping density increases, as shown in Fig. 5. This can be understood from the following discussion. The optimum dark current level, where the responsivity or the detectivity becomes maximum, exists for each doping density. Responsivity and detectivity are maximum at a specific bias voltage, respectively, ${ }^{1,13}$ ) since quantum efficiency and photoconductive gain also does. ${ }^{14,15)}$ This denotes that there are an optimum operation voltage and an optimum dark current. The dark current is known to be detrimental to the performance of QDIPs. However, it also has a positive aspect. This is because the dark current is necessary to refill QDs with electrons and to have a photoconductive gain. ${ }^{16)}$ Therefore, an optimum dark current level always exists. The optimum dark current level is not dependent on the doping density, since the detectivity is not a strong function of the doping density as discussed above. ${ }^{12)}$ Figures 5 and 6 show these tendencies experimentally: the maximum detectivity does not depend on the doping density and the optimum dark current level is $\sim 5 \mathrm{~mA}$ (current density: $\sim \mathrm{A} / \mathrm{cm}^{2}$ ) for the samples regardless of the doping density. On the other hand, as shown in Fig. 6, the dark current depends very sensitively on the applied bias voltage as well as on the doping density, that is, it increases exponentially with increasing doping density or bias voltage. ${ }^{12,17)}$ As a result, as the doping density increases, the dark current reaches the optimum level with smaller bias voltage, and the optimum operation voltage becomes smaller.

Peak responsivities and detectivities of the samples as functions of temperature are shown in Fig. 7. The possibility of high-temperature operation of QDIP is confirmed from clear IR responses up to $180 \mathrm{~K}$ for all the samples, although the response peak wavelength becomes shorter as temper-

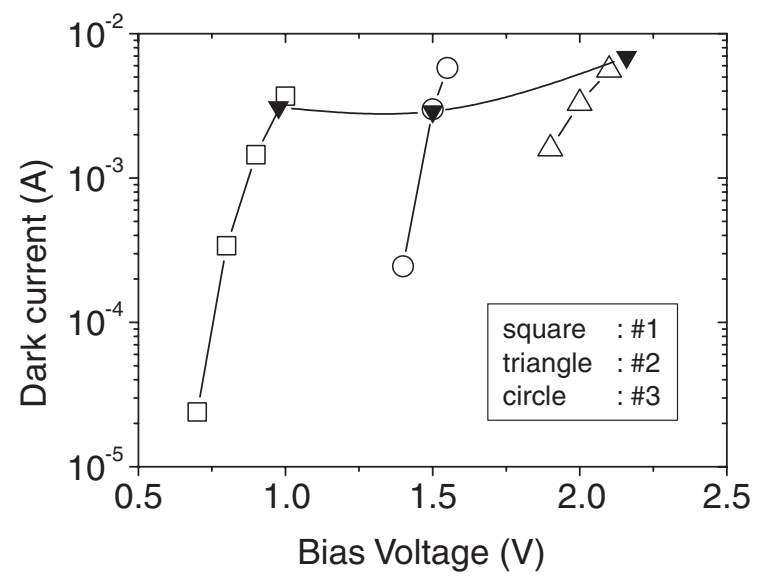

Fig. 6. Dark currents at each measurement bias voltage of each QDIP (open symbols) and optimum dark current level (solid symbols). The optimum level of dark current is almost independent of the doping density in the range used in this experiment $\left(5 \times 10^{16}-5 \times 10^{17} / \mathrm{cm}^{3}\right)$.

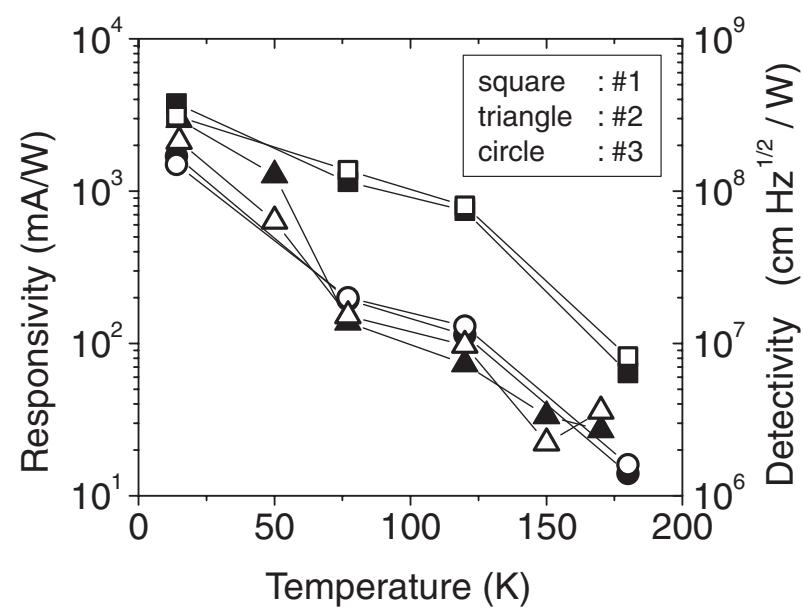

Fig. 7. Temperature-dependent peak responsivities (solid symbols) and detectivities (open symbols).

ature increases. QDIPs certainly operate at a temperature higher than that of QWIPs. This is partly due to the large photoconductive gain. ${ }^{3)}$ In addition, responsivity and detectivity of each sample show similar behaviors with respect to temperature, since the optimum dark current behavior with temperature is very similar for each sample.

The temperature-dependent behaviors of responsivity and detectivity of each sample, as shown in Figs. 7 and 5, are improved as the doping density increases in the range used in this experiment $\left(5 \times 10^{16}-5 \times 10^{17} / \mathrm{cm}^{3}\right)$. As a reference sample, the undoped QDIP with the same structure operates at less than $100 \mathrm{~K}$. The number of electrons in QDs must be also important for the operation of QDIP. The number of electrons in QDs at a given temperature increases with the doping density since the thermal activation rate of electrons in QDs remains the same with different doping densities. Therefore, the doping density should be higher than that of QDIP \#1 in order to realize the high-temperature operation of QDIP. This, of course, will be applicable only when the QD quality is not degraded with the increase in doping density. The PL data show that modulation doping does not degrade the QD quality.

\section{Summary}

The performance of QDIPs was investigated as a function of doping density. We found that the optimum operation voltage decreases with increasing doping density. This is because the optimum dark current level is almost independent of the doping density. We confirmed experimentally that the optimum dark current level is $\sim 5 \mathrm{~mA}$ (current density: $\sim \mathrm{A} / \mathrm{cm}^{2}$ ) for the samples regardless of the doping density. It was found that it is necessary to increase the doping density more, since the detectivity and responsivity with respect to temperature were improved with increasing the doping density. The possibility of higher temperature operation of QDIP and responses to normal incident light were also demonstrated.

\section{Acknowledgments}

This work was supported, in part, by KISTEP (under IMT2000 R\&D donation support program) and MOE BK21 programs. 
1) D. Pan, E. Towe and S. Kennerly: Appl. Phys. Lett. 75 (1999) 2719.

2) S.-F. Tang, S.-Y. Lin and S.-C. Lee: Appl. Phys. Lett. 78 (2001) 2428.

3) L. Jiang, S. S. Li, N.-T. Yeh, J.-I. Chyi, C. E. Ross and K. S. Jones: Appl. Phys. Lett. 82 (2003) 1986.

4) N. Horiguchi, T. Futatsugi, Y. Nakata, N. Yokoyama, T. Mankad and P. M. Petroff: Jpn. J. Appl. Phys. 38 (1999) 2559.

5) Y. S. Kim, U. H. Lee, D. Lee, S. J. Rhee, Y. A. Leem, H. S. Ko, D. H. Kim and J. C. Woo: J. Appl. Phys. 92 (2002) 7462.

6) R. D. Hudson: Infrared System Engineering (John Wiley \& Sons, New York, 1969).

7) E. M. Daly, T. J. Glynn, J. D. Lambkin, L. Considine and S. Walsh: Phys. Rev. B 52 (1995) 4696.

8) B. F. Levine, A. Zussman, S. D. Gunapala, M. T. Asom, J. M. Kuo and W. S. Hobson: J. Appl. Phys. 72 (1992) 4429.

9) U. H. Lee, D. Lee, H. G. Lee, S. K. Noh, J. Y. Leem and H. J. Lee: Appl. Phys. Lett. 74 (1999) 1597.

10) B. Kochman, A. D. Stiff-Roberts, S. Chakrabarti, J. D. Phillips,
S. Krishna, J. Singh and P. Bhattacharya: IEEE J. Quantum Electron. 39 (2003) 459.

11) Z. Ye, J. C. Campbell, Z. Chen, E.-T. Kim and A. Madhukar: Appl. Phys. Lett. 83 (2003) 1234.

12) S. D. Gunapala, B. F. Levine, L. Pfeiffer and K. West: J. Appl. Phys. 69 (1991) 6517.

13) S. J. Xu, S. J. Chua, T. Mei, X. C. Wang, X. H. Zhang, G. Karunasiri, W. J. Fan, C. H. Wang, J. Jiang, S. Wang and X. G. Xie: Appl. Phys. Lett. 73 (1998) 3153.

14) Z. Ye, J. C. Campbell, Z. Chen, E.-T. Kim and A. Madhukar: J. Appl. Phys. 92 (2002) 7462.

15) U. H. Lee, Y. H. Kang, J. H. Oum, S. Hong, S. Kang, S. Lee, M. Kim and S. K. Noh: submitted to J. Appl. Phys.

16) H. C. Liu: Appl. Phys. Lett. 60 (1992) 1507

17) V. Ryzhii, V. Pipa, I. Khmyrova, V. Mitin and M. Willander: Jpn. J. Appl. Phys. 39 (2000) L1283. 